\title{
Molecular dynamics simulation on glass transition temperature of isomeric polyimide
}

\author{
M. Li, X. Y. Liu, J. Q. Qin, Y. Gu* \\ State Key Laboratory of Polymer Material and Engineering, College of Polymer Science and Engineering, Sichuan Uni- \\ versity, Chengdu 610065, China
}

Received 5 June 2009; accepted in revised form 12 August 2009

\begin{abstract}
It is an abnormal phenomenon that glass transition temperature $\left(T_{g}\right)$ of isomeric polyimide (PI) is higher than its corresponding symmetrical PI. To illustrate this phenomenon at the molecular scale, we applied molecular dynamics method to predict the $T_{g}$ of PI, which were prepared based on 4,4'-oxydianiline (ODA) and 3,3',4,4'-biphenyltetracarboxylic dianhydride (3,3',4,4'-BPDA), and its isomeric system (2,2',3,3'-BPDA-ODA). Simulation result is consistent with experimental value. Non-bond energy plays an important role in glass transition process, for it has an abrupt change near $T_{g}$. The higher free volume fraction of isomeric PI can provide the polymer with more space to obtain segmental motion. However, from the torsion angle distribution calculations, it is shown that the torsion angle of its biphenyl group is constrained. Furthermore, from mean square displacement and vector autocorrelation functions calculations, this group is observed to rotate against other groups in the glassy state, and increases the chain rigidity to a great extent. So the isomeric PI needs much more relaxation time for the segment motion. Therefore, the higher $T_{g}$ of isomeric PI is mainly attributed to the chain rigidity for the time scale, not the free volume for the space scale.
\end{abstract}

Keywords: modeling and simulation, isomeric polyimide, molecular dynamics simulation, glass transition temperature

\section{Introduction}

Glass transition temperature $\left(T_{g}\right)$ is a key index to evaluate the thermal properties of heat-resistant polymers, such as polyimide (PI), polysulfone, polyether ether ketone. As for polyimide, isomeric PI, which incorporate geometrically asymmetrical unit in dianhydride or diamine structures, can improve the processability and solubility; at the same time, its thermal properties do not show obvious decrease for there is no composition change compared with corresponding symmetrical PI. Unexpectedly, the $T_{g}$ of isomeric PI is relatively higher than that of symmetrical PI [1]. This interesting phenomenon is not observed with other polymers, such as polyamide [2] and poly(arylene ether ketone) [3, 4]. Several researchers have tried to explain this phenomenon: Tong et al. [5] reported that the $T_{g}$ of symmetrical PI, which was polymerized from $3,3^{\prime}, 4,4^{\prime}$ 'biphenyltetracarboxylic dianhydride (3,3',4,4'-BPDA) and 4,4'oxydianiline (ODA), was $52 \mathrm{~K}$ lower than that of its isomeric system (2,2',3,3'-BPDA-ODA). Furthermore, they analyzed the relative energies of torsion angle for PI monomer, and suggested that the steric hindrance of the adjacent anhydride groups of 2,2',3,3'-BPDA inhibit the segmental motion, and increase the $T_{g}$, relative to that of the 3,3',4,4'BPDA. Kochi et al. [6] reported similar results on these two systems, and analyzed the temperature of $\beta$ relaxation using the dynamic mechanical analysis (DMA) method. Hasegawa et al. [7] studied on the isomeric phenomena of PIs, which were prepared 
based on BPDA and 1,4-phenylene diamine (PDA). Semiempirical method was used to study the conformation of a single PI chain in gas state. They inferred that the higher $T_{g}$ for the isomeric polyimide is attributed to the asymmetric structure requiring a larger sweep volume for the crank shaft motion and accordingly more energy (higher temperature) to obtain this motion. The above studies facilitate us with the insight into the nature of the higher $T_{g}$ of isomeric PI, however, there has been no systematic study investigating the effect of isomerism on $T_{g}$ of polyimides. It is acknowledged that the $T_{g}$ of polymeric materials is mainly determined by the segment motion of a polymer chain. If this motion can be observed, the polymer chain should have sufficient space (more free volume) and can complete this motion in a short time period (less relaxation time, it mainly depends on rigidity of a polymer chain). Nevertheless, these two factors can not be well discussed in the gas state [5, 7], so it is necessary to find an appropriate method to investigate them in the bulk state.

Yet, molecular dynamics (MD) simulation method provides a proper means to study bulk polymer at molecular scale. Some researchers have applied it to study the glass transition temperature of polymers, such as polyethylene [8], polypropylene [9], polystyrene [9], poly(oxymethylene) [8], polysiloxane [10], polylactic acid [11], epoxy resin [12]. The results indicate a good agreement with experimental value. In the filed of polyimides, Liang et al. [13] successfully predict the glass transition temperature of PI with different chromophores using MD method, and they deduced that $T_{g}$ of PIs are correlated to the height of energy barrier of bridging bonds between aromatic rings. Recently, Yani and Lamm [14] studied on the glass transition temperature of $\mathrm{PI} /$ polyhedral oligomeric silsesquioxane (POSS) blends, showing that the simulation results are comparable to experimental observation and incorporation of the appropriate content POSS can improve glass transition temperature. Thus, MD method is an effective method to predict the glass transition temperature of polymers at the molecular scale.

In this study, we firstly applied MD simulation method to study the effect of isomerism on the glass transition temperature of bulk polyimides, using 3,3',4,4'-BPDA-ODA and 2,2',3,3'-BPDAODA systems (Figure 1) as model molecules. The

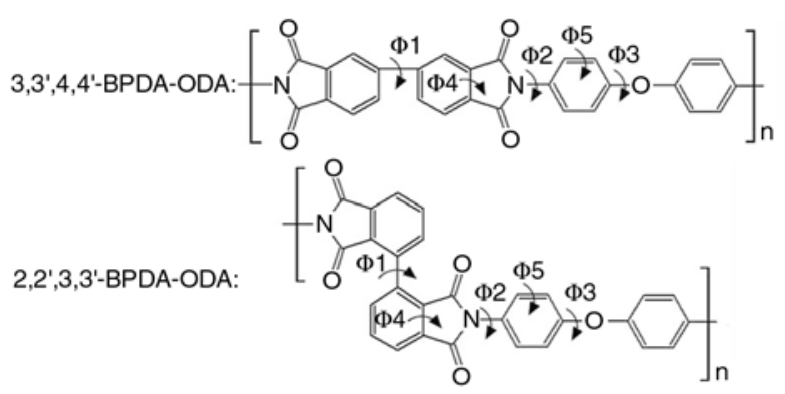

Figure 1. Structures of two polyimides: 3,3',4,4'-BPDAODA and 2,2',3,3'-BPDA-ODA

rest of the paper is organized as follows: In section 2, simulation details of MD method are described. Section 3 is result and discussion. We obtained comparative value of $T_{g}$ in PIs, and analyze the role of energy component in glass transition process. Furthermore, the reason for higher $T_{g}$ of 2,2',3,3'-BPDA-ODA systems is given by the analysis at time scale (chain rigidity) and space scale (free volume). Finally, we summarize our major results and conclusions in section 4 .

\section{Simulation details}

Simulations were performed using the Materials Studio 4.0 software purchased from Accelrys Inc [15]. The Dreiding force field was used in molecular mechanics (MM) and molecular dynamics simulations [16]. The total potential energy $\left(E_{\text {total }}\right)$ can be expressed as:

$$
\begin{aligned}
E_{\text {total }}= & E_{\text {bond }}+E_{\text {non-bond }}= \\
& E_{b}+E_{\theta}+E_{\varphi}+E_{\text {inv }}+E_{v d W}+E_{\text {elec }}
\end{aligned}
$$

Equation (1) is divided into two parts: valence terms $\left(E_{\text {bond }}\right)$ and non-bond interaction terms $\left(E_{\text {non-bond }}\right)$. The valence terms include $E_{b}, E_{\theta}, E_{\varphi}$, $E_{i n v}$ for bond stretching, angle bending, dihedral torsion and inversion (out-of-plane) terms. The non-bond interaction terms include $E_{v d W}$ and $E_{\text {elec }}$ for calculating van der Waals force and electrostatic interactions respectively. The non-bond interactions were calculated in this study with cut-off being set to $0.95 \mathrm{~nm}$, which is less than half of the cell length (during the glass transition process, cell length was kept around 2.2-2.5 nm). The amorphous polymer models were built with the Theodorou and Suter's method $[17,18]$ using periodic boundary conditions. Each cell consisted of a parent PI chain with 20 repeat units. Though the system size of 20 repeat units is not enough to rep- 
resent the conformation of a real polymer chain, previous researchers have reported reasonable result when they used 10-15 repeat units $[13,19]$. In addition, previous studies demonstrate that simulated $T_{g}$ values are comparable with experimental one by simulating a single polymer chain $[8-10$, 13]. So we can save a lot of computational resource in this way. The cell was first optimized in a low initial density of $0.5 \mathrm{~g} / \mathrm{cm}^{3}$ by smart minimization, which incorporated steepest decent with conjugate gradient methods. Then, relaxation of the cell was performed by MD simulation at $1000 \mathrm{~K}$ for $2 \mathrm{~ns}$ using an integration step of $1 \mathrm{fs}$ under constantpressure constant-temperature (NPT) ensemble. The conformation of low potential energy structure was selected and minimized again. In this procedure, density of each PI system was above $0.8 \mathrm{~g} / \mathrm{cm}^{3}$. To make sure a system reached equilibrium, a number of thermodynamic quantities were checked. As a result, they remained stable during the last $500 \mathrm{ps}$, and it was concluded that the system was equilibrated.

The glass transition temperature of polymeric materials can be determined by plotting specific volume versus temperature with constant-NPT MD simulation and noting where the slope changes [14]. The system was cooled from $750 \mathrm{~K}$ stepwise to $400 \mathrm{~K}$ by decrements of $50 \mathrm{~K}$. At each temperature, constant-NPT MD simulation was conducted for $1 \mathrm{~ns}$. In the last $100 \mathrm{ps}$, the cell density was sampled at every picosecond, and the average value was taken as the result.

In addition to the glass transition temperatures, we have also calculated torsion angle distribution, mean square displacement and vector autocorrelation functions of polyimides. The equilibrated configuration in the constant-NPT MD simulation was used as the initial structure, and then was calculated by constant-volume constant-temperature (NVT) MD simulation for $2 \mathrm{~ns}$. Data samples were collected at every picoseconds.

In this study, the pressure of system was set to $1.0 \cdot 10^{5} \mathrm{~Pa}$. Nose thermostat $[20,21]$ and Berendsen barostat [22] were applied to control temperature and pressure in constant-NPT MD simulation.

\section{Results and discussion}

\subsection{Glass transition temperature}

The temperature dependence of the specific volume of two PI systems is shown in Figure 2. With increasing temperature, the increment of specific volume is less pronounced below $T_{g}$ yielding a kink in the curve to determine $T_{g}$. Detailed method has been well described in ref. [11]. According to this method, simulated glass transition temperatures of these two PIs were obtained. The $T_{g}$ of isomeric PI $\left(2,2^{\prime}, 3,3^{\prime}\right.$-BPDA-ODA) is $593 \mathrm{~K}$ which is much higher than symmetrical PI's (3,3',4,4'-BPDAODA, $548 \mathrm{~K})$. Simulation result is consistent with experimental values shown in Table 1. For comparison, we also applied group contribution method to predict $T_{g}$ of PIs [13]. This method is based on the contribution of each functional group to thermodynamic properties of polymeric materials, and it has been widely used in alkyl polymers [23, 24]. However, in this study, the $T_{g}$ values of two PIs are the same using this method for it can not resolve the position effect of the imide group. Therefore, MD simulation is a relatively effective method to pre$\operatorname{dict} T_{g}$ of isomeric polyimides.

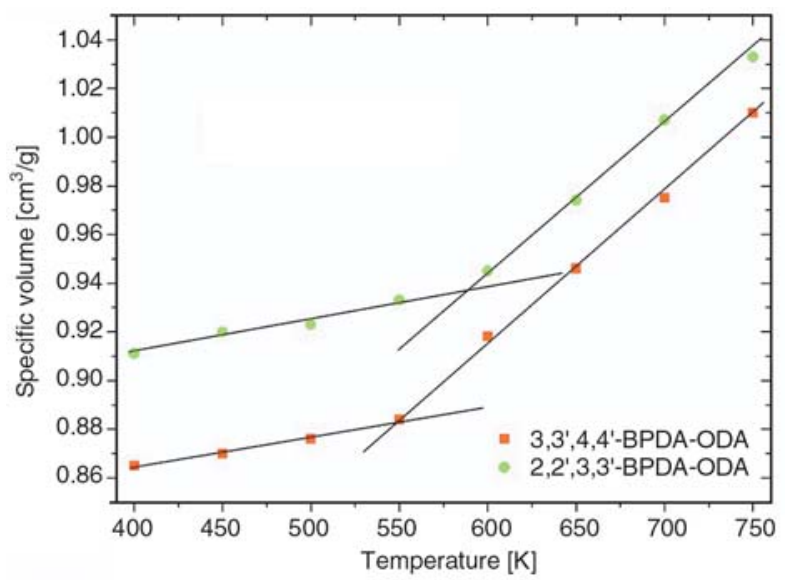

Figure 2. Plots of specific volume of MD simulation versus temperature for two polyimides: 3,3',4,4'BPDA-ODA ( $\bullet) ; 2,2^{\prime}, 3,3^{\prime}-B P D A-O D A ~(\bullet)$

Table 1. Glass transition temperatures of PIs with MD simulation, correlation prediction, and experiment methods

\begin{tabular}{|c|c|c|c|}
\hline \multirow{2}{*}{ PI systems } & \multicolumn{3}{|c|}{ Glass transition temperature [K] } \\
\cline { 2 - 4 } & MD simulation & Correlation prediction $^{\mathbf{a}}$ & Experiment $^{\mathbf{b}}$ \\
\hline $3,3^{\prime}, 4,4^{\prime}$ '-BPDA-ODA & 548 & 576 & 539 \\
\hline $2,2^{\prime}, 3,3^{\prime}$-BPDA-ODA & 593 & 576 & 591 \\
\hline
\end{tabular}

aPredicted by Synthia module in Materials Studio software package

${ }^{b}$ Experimental value in ref. [5] 
Also, we checked the density of PIs at $298 \mathrm{~K}$. The density for 3,3',4,4'-BPDA-ODA system is $1.20 \mathrm{~g} / \mathrm{cm}^{3}$ while the density of $2,2^{\prime}, 3,3^{\prime}$-BPDAODA system is $1.13 \mathrm{~g} / \mathrm{cm}^{3}$. And the density of $3,3^{\prime}, 4,4^{\prime}$-BPDA-ODA system is about $17 \%$ smaller than experiment value [25] (density of 2,2',3,3'BPDA-ODA system has not been reported in existing reference). However, when we want to get the comparable $T_{g}$ from the MD simulation, the dynamic change of density (or specific volume) near $T_{g}$ should be emphasized, not the density itself.

\subsection{Free volume}

According to the Fox and Flory's theory of glass transition, the free volume of the polymer experiences an abrupt change during the cooling down at a point called the glass-transition temperature. In this study, we applied a probe sphere, whose radius is $0.1 \mathrm{~nm}$ (distance between neighboring atoms) or $0.3 \mathrm{~nm}$ (distance between neighboring molecules), to probe the equilibrated polymer cells to calculate the free volume [8]. Furthermore, the free volume fraction (FVF) can be obtained by Equation (2):

$$
F V F=\frac{V_{f}}{V_{f}+V_{0}}
$$

where $V_{f}$ is free volume, and $V_{0}$ is occupied volume of the polymer chains.

The plot of FVF of two PIs with a probe sphere of $0.1 \mathrm{~nm}$ radius versus temperature is shown in Figure 3, indicating similar $T_{g}$ with the plot of specific

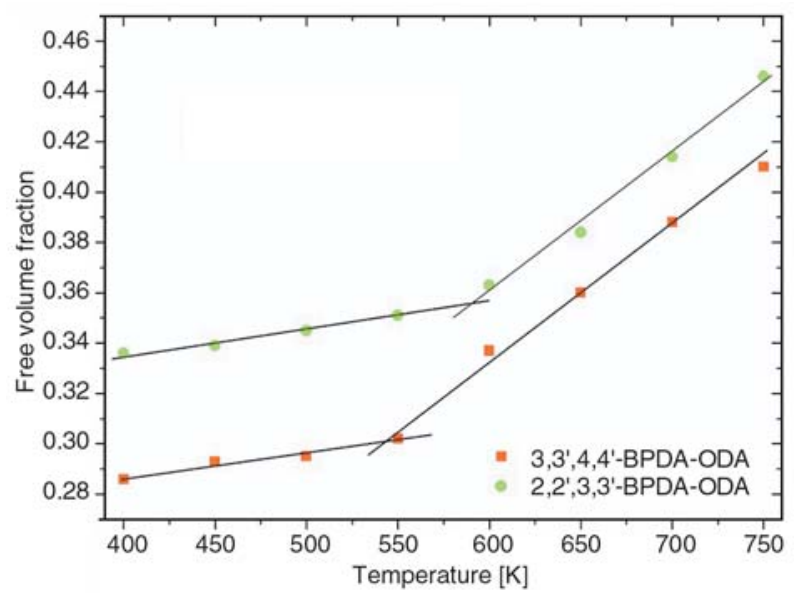

Figure 3. Plots of free volume fraction versus temperature for two polyimides: 3,3',4,4'-BPDA-ODA ( $($ ) 2,2',3,3'-BPDA-ODA (•) volume versus temperature. However, increasing the radius to $0.3 \mathrm{~nm}$, we can not get the clear $T_{g}$ by the same method, for it is difficult to determine van der Waals surface with the probe sphere of big radius in this measurement. During the glass transition process, free volume determines how much space can be provided for the segment motion of a polymer chain. For polymers with similar compositions, the lower FVF can bring with higher $T_{g}$ of that polymeric materials, for it can not provide sufficient space for segment motion at the same temperature. It is clear that free volume fraction of $2,2^{\prime}, 3,3^{\prime}$-BPDA-ODA system is higher than $3,3^{\prime}, 4,4^{\prime}$-BPDA-ODA system at the same temperature, as the same result for specific volume showing in Figure 2. In 2,2',3,3'-BPDA-ODA system, incorporation of asymmetry group decreases the regularity of polymer chain, inhibits the close packing of inter polymer chain, and increases free volume at molecular scale, namely facilitates the segment motion to some extent. This result may go against the result of higher $T_{g}$ of isomeric PIs. However, free volume is not the single variable to evaluate the $T_{g}$ of PIs, other factors that may affect the $T_{g}$ will be discussed in the following sections.

\subsection{Role of the energy components in glass transition process}

To understand the role of each energy component playing in glass transition process, we examined the plot of the energy component versus temperature for two PI systems, and the result shows that the PIs show the same trend in the plot. Take isomeric 2,2',3,3'-BPDA-ODA system for example, the simulated results of bond energy, angle energy, torsion energy, inversion energy and non-bond energy versus the temperature were plotted in Figure 4. There is a kink only in the plot of non-bond energy versus temperature, indicating the occurrence of glass transition. At both below and above $T_{g}$, non-bond energy increases almost linearly with increasing temperature with a break at $T_{g}$. But for the other energy components, they consistently increase linearly with increasing temperature in the whole temperature range. These results show that the non-bond energy plays an important role in the glass transition process of the polymer system. The above results are consistent with the same analysis on epoxy resin by $\mathrm{Wu}$ and $\mathrm{Xu}$ [12]. However, $\mathrm{Li}$ 

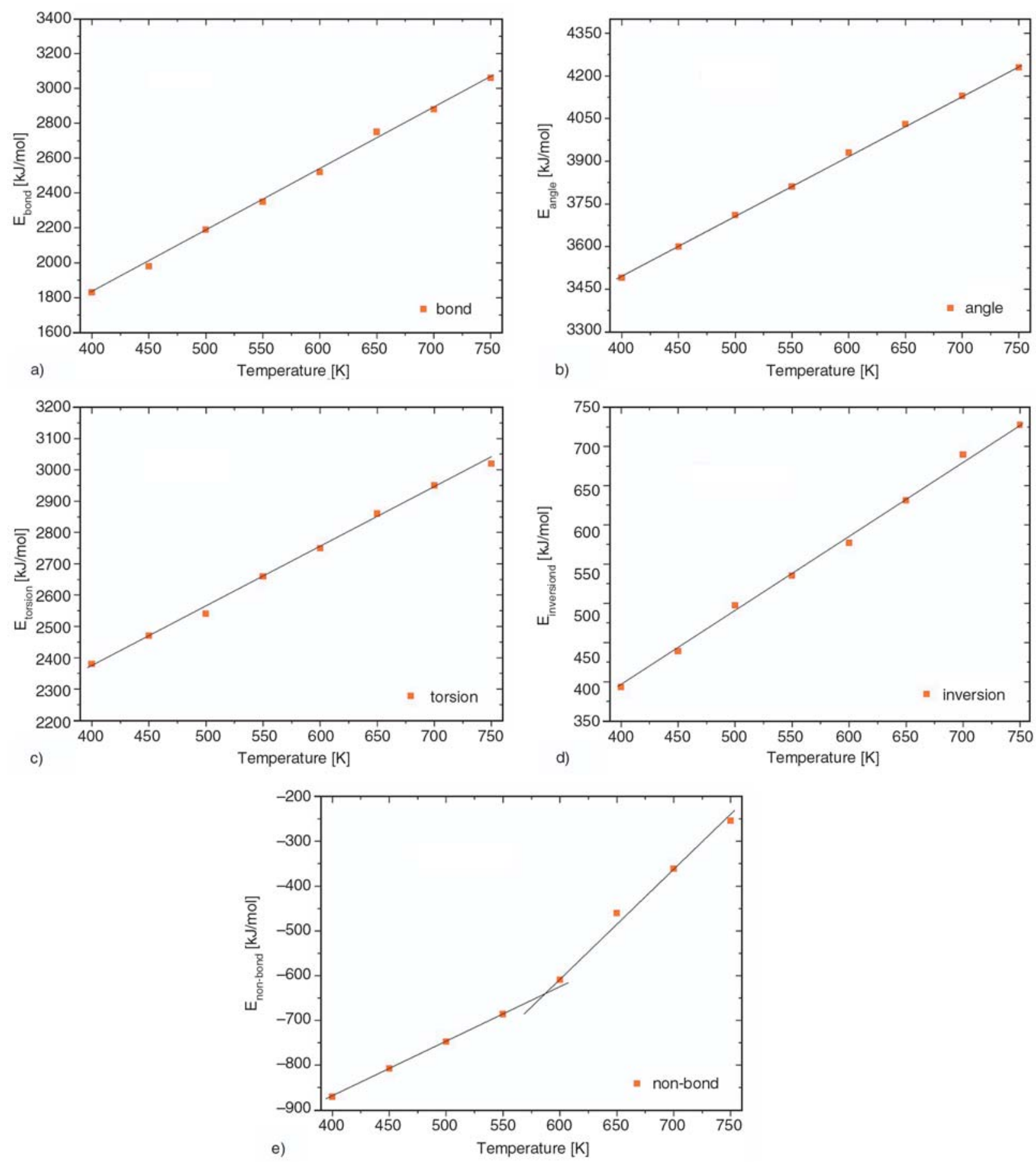

Figure 4. Plots of some energy components versus temperature of 2,2',3,3'-BPDA-ODA: (a) bond energy; (b) angle energy; (c) torsion energy; (d) inversion energy; (e) non-bond energy

and coworkers $[8,26]$ also performed the same analysis on polyoxymethylene (POM) system [8] and poly(3-hydroxybutyrate) (PHB)/poly(ethylene oxide) (PEO) blend system [26] and drew a different conclusion from ours: both torsion and nonbond energy played important roles in glass transition. In fact, the freedom of bond stretching and angle bending could be equilibrated fast in the simulation, therefore the linear increasing trend are shown. Unlike the common alkyl polymers, aromatic PI has much higher $T_{g}$ for it has plenty of phenyl and imide rings, so the dihedral torsion and out-of-plane motion of aromatic PIs are constrained, and no kink has been observed in this study. However, the segment motion ability of aromatic PIs are mainly attributed to the limited torsion of linking of phenyl and imide rings [13]. Influenced by the thermal fluctuation, limited link- 
ing groups show distinct mobility, yielding the conformation transition, and furthermore, resulting in the sudden change of non-bond energy near $T_{g}$.

\subsection{Torsion angle distribution}

As discussed in section 3.3, mobility of linking groups exerts an important influence during the glass transition process. In order to elucidate the reason for the higher $T_{g}$ of isomeric PI, torsion angle distributions of linking in two PIs were plot-
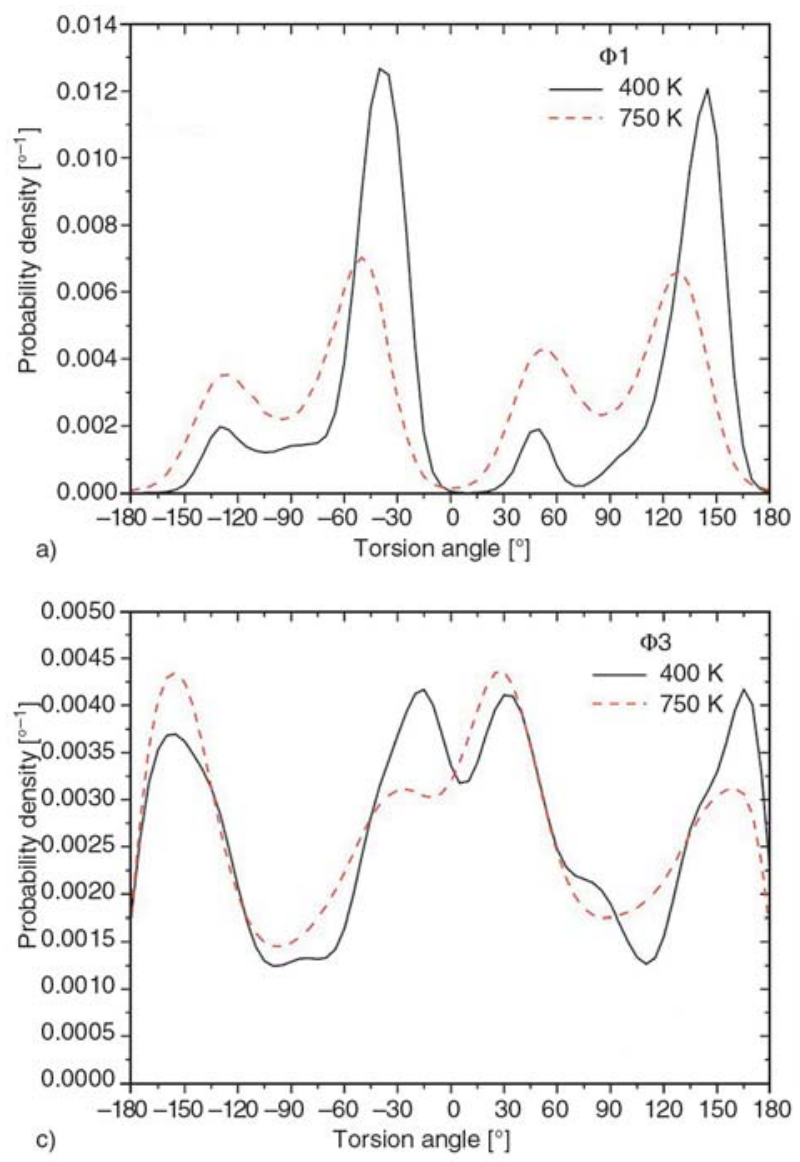

ted (Figure 5 and Figure 6). Torsion angles $\Phi 1, \Phi 2$ and $\Phi 3$ were defined in Figure 1. For facilitating the discussion, we defined $\mathrm{C}_{\mathrm{p}}$ for carbon atom in phenyl ring and $\mathrm{C}_{\mathrm{i}}$ for carbon atoms in imide ring linking the ether group. Therefore, we can define $\mathrm{C}_{\mathrm{p}}-\mathrm{C}_{\mathrm{p}}-\mathrm{C}_{\mathrm{p}}-\mathrm{C}_{\mathrm{i}}(\Phi 4)$ as the torsion angle of $\mathrm{C}_{\mathrm{p}}-\mathrm{C}_{\mathrm{p}}$ between phenyl and imide rings, and $\mathrm{H}-\mathrm{C}_{\mathrm{p}}-\mathrm{C}_{\mathrm{p}}-\mathrm{C}_{\mathrm{p}}$ (Ф5) as torsion angle of $\mathrm{C}_{\mathrm{p}}-\mathrm{C}_{\mathrm{p}}$ in the phenyl ring which is between imide ring and ether group. Furthermore, the $\Phi 4$ and $\Phi 5$ can be used for study of the co-plane properties of these aromatic rings. As
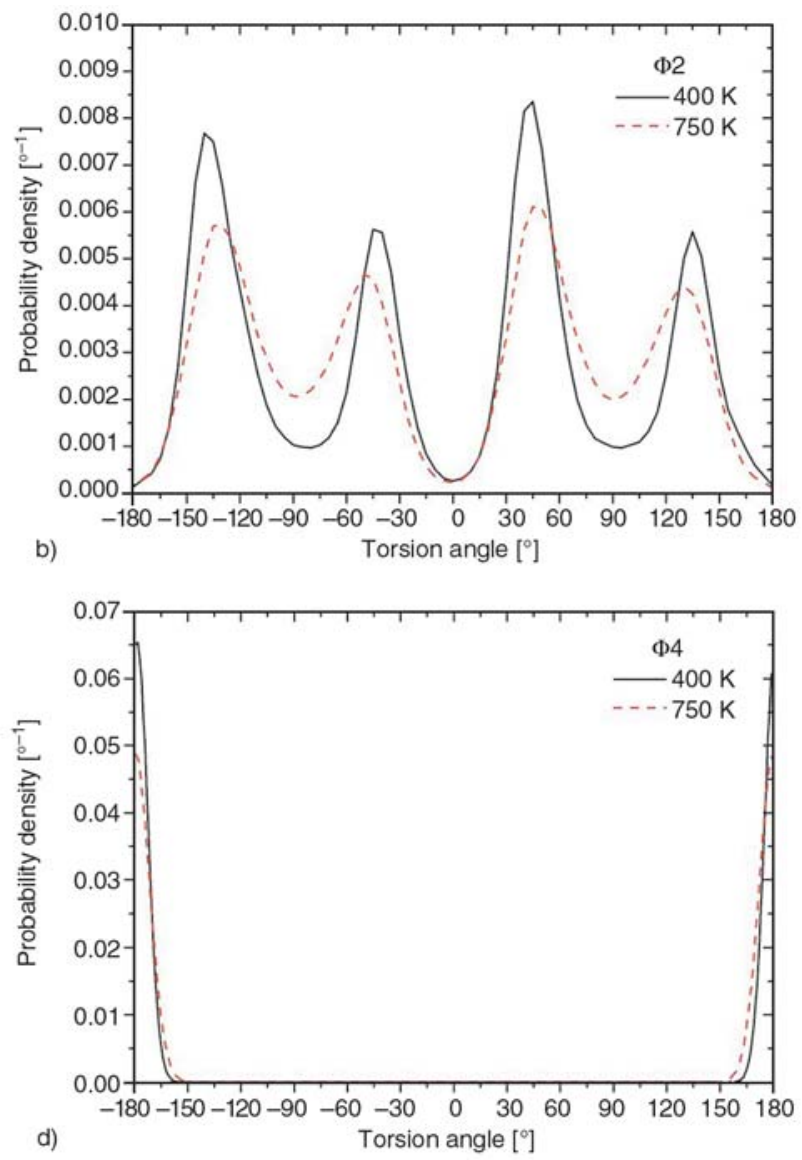

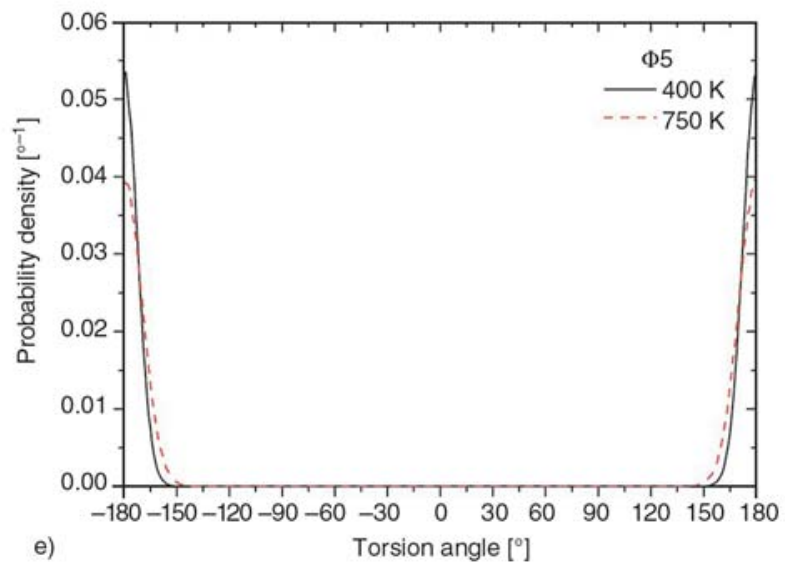

Figure 5. Torsion angle distributions of 3,3',4,4'-BPDA-ODA: (a) $\Phi 1$, (b) $\Phi 2$, (c) $\Phi 3$, (d) $\Phi 4$ and (e) $\Phi 5$. The results have been obtained with constant-NVT MD simulation at $T=400 \mathrm{~K}\left({ }_{-}\right)$and $750 \mathrm{~K}(--)$. 

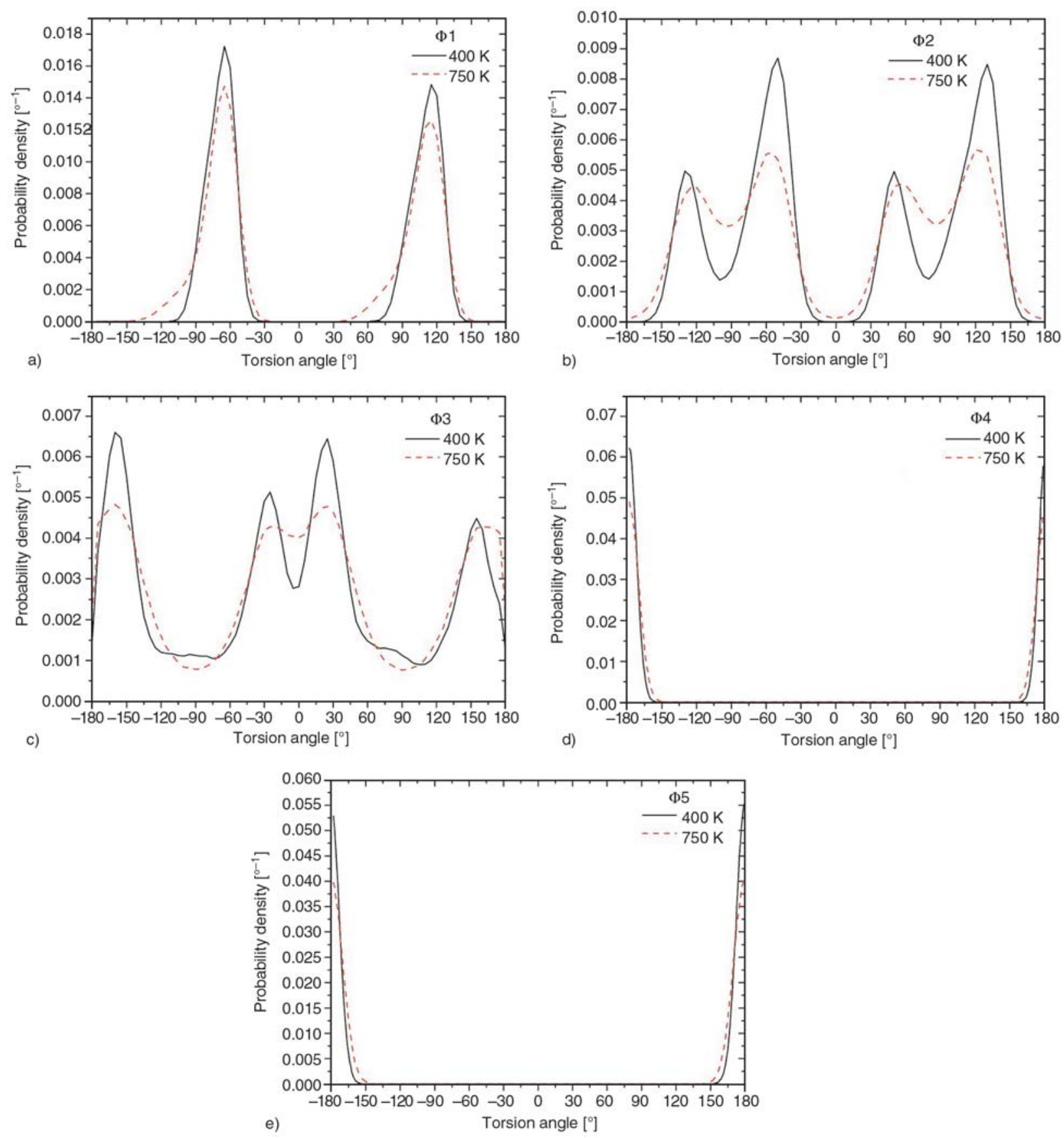

Figure 6. Torsion angle distributions of 2,2',3,3'-BPDA-ODA: (a) $\Phi 1$, (b) $\Phi 2$, (c) $\Phi 3$, (d) $\Phi 4$ and (e) $\Phi 5$. The results have been obtained with constant-NVT MD simulation at $T=400 \mathrm{~K}(-)$ and $750 \mathrm{~K}(--)$.

for $\Phi 1$ (torsion angle of $\mathrm{C}_{\mathrm{p}}-\mathrm{C}_{\mathrm{p}}$ between phenyl ring), 3,3',4,4'-BPDA-ODA system has peaks in $\pm 35^{\circ}$ and $\pm 135^{\circ}$, but in isomeric $2,2^{\prime}, 3,3^{\prime}$-BPDAODA system, there are only two peaks located in the $\pm 60^{\circ}$. This result is similar to the plot of relative energy of torsion angles for PI monomers in the same systems obtained by molecular mechanics in the previous study [5]. Higher rotational barrier corresponding to $\Phi 1$ in $2,2^{\prime}, 3,3^{\prime}$-BPDA-ODA system is responsible for the two symmetrical two peaks in the torsion angle distribution, and eventu- ally results in its higher $T_{g}$. Two PIs have the same peak position but different relative proportion for $\Phi 2$ (torsion angle of $\mathrm{N}-\mathrm{C}_{\mathrm{p}}$ between imide and phenyl rings) and $\Phi 3$ (torsion angle of $\mathrm{C}_{\mathrm{p}}-\mathrm{O}$ in diamine structure). As for $\Phi 4$ and $\Phi 5$, two PIs have only two half peaks in $\pm 180^{\circ}$, illustrating that phenyl and imide ring can keep in a plane well under the premise of phenyl ring's keeping in a plane. To explore the effect of temperature on torsion angle distribution, two PIs' torsion angle distributions in 400 and $750 \mathrm{~K}$ were given. These 
distribution functions exhibit peak at the same position at two different temperatures, only a wide distribution at the higher temperature, especially $\Phi 2$ and $\Phi 3$. No matter at glassy or rubbery state, the torsion angles of $\Phi 4$ and $\Phi 5$ are kept around $\pm 180^{\circ}$. It proves that atoms in phenyl and imide ring of PIs can be kept in a plane respectively during the glass transition process. Furthermore, it also confirms that the torsion energy does not have sudden change in the glass transition process due to confined torsion, which has been discussed in section 3.3 .

\subsection{Mean square displacement}

The value of $T_{g}$ is correlated well with the polymer rigidity, namely the segmental mobility in polymer chain. To observe the mobility of PI chains during glass transition process, the mean square displacements (MSD) were calculated [14]. With the increasing temperature, the slope of MSD of both two PI systems becomes steeper. Take 2,2',3,3'BPDA-ODA system as an example (Figure 7), the slope of MSD above $T_{g}(700$ and $750 \mathrm{~K})$ are much higher than that below $T_{g}(400$ and $500 \mathrm{~K})$, indicating higher mobility of PI chains above $T_{g}$. Furthermore, we have also compared the mobility of 3,3',4,4'-BPDA-ODA and 2,2',3,3'-BPDA-ODA systems. Figure 8 shows the mean square displacement plot for the two PI systems at 400 and $750 \mathrm{~K}$. It is shown that the MSD for 2,2',3,3'-BPDA-ODA system is lower than that for 3,3',4,4'-BPDA-ODA system at all times. This means $2,2^{\prime}, 3,3^{\prime}$ 'BPDAODA system has lower mobility than $3,3^{\prime}, 4,4^{\prime}$ BPDA-ODA system, and hence it needs more time

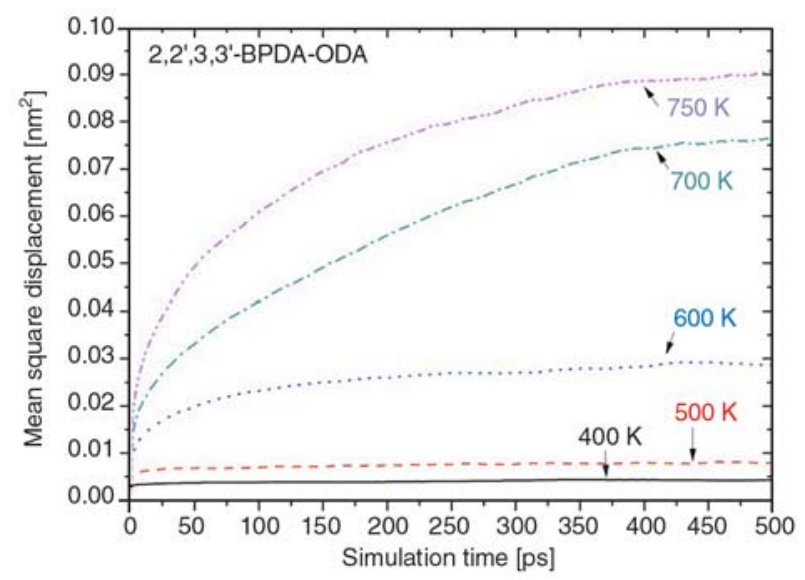

Figure 7. Mean square displacements of 2,2',3,3'-BPDAODA system as a function of temperature

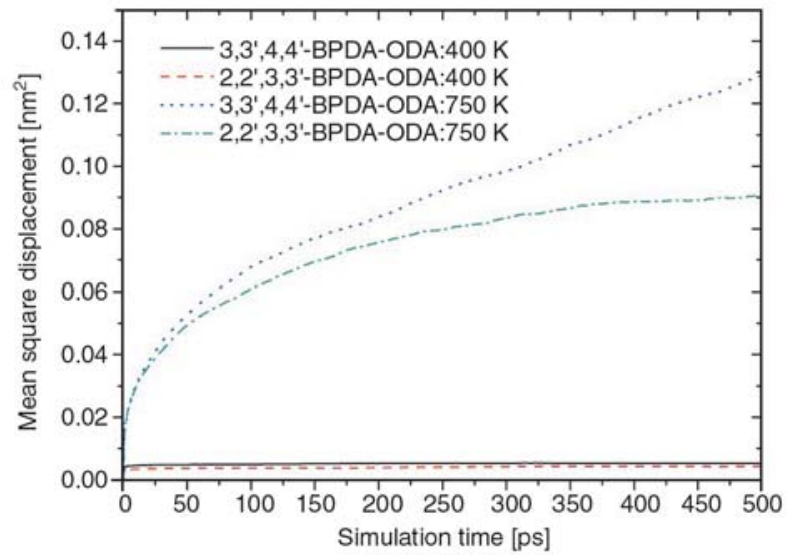

Figure 8. Mean square displacements of 3,3',4,4'-BPDAODA and 2,2',3,3'-BPDA-ODA systems at $T=400 \mathrm{~K}$ and $750 \mathrm{~K}$

(or higher temperature) to obtain segment motion. The above observations support the result of higher $T_{g}$ of 2,2',3,3'-BPDA-ODA systems.

\subsection{Vector autocorrelation function}

To further characterize the mobility of PI chains, the mobility of linking groups were examined. It can be quantified by vector autocorrelation functions (VACFs) of these groups along the polymer chain (Equation (3)):

$M_{2}(t)=\frac{3<(u(t) \bullet u(0))^{2}>-1}{2}$

where $u(t)$ and $u(0)$ represent unit vectors of the segment at time $t$ and time 0 , respectively. The $<>$ indicates an ensemble average over such vectors [12]. At the same simulation time, the higher value of $M_{2}(t)$ indicates the higher rigidity of the segment.

The segmental vectors corresponding to the torsion angle $\Phi 1, \Phi 2, \Phi 3, \Phi 4$ and $\Phi 5$ are shown in Table 2. They are defined as the vectors from the first atom to the fourth atom of the torsion angles. The plots of VACFs of these segments in two PIs are given at two temperatures: $400 \mathrm{~K}$ (Figure 9) and $750 \mathrm{~K}$ (Figure 10) [12]. At $400 \mathrm{~K}$, the value

Table 2. Definition of segmental vectors corresponding to torsion angle $\Phi 1, \Phi 2, \Phi 3, \Phi 4$ and $\Phi 5$

\begin{tabular}{|c|c|c|}
\hline Torsion angle & Atoms & Segmental vector \\
\hline$\Phi 1$ & $\mathrm{C}_{\mathrm{p} 1}-\mathrm{C}_{\mathrm{p} 2}-\mathrm{C}_{\mathrm{p} 3}-\mathrm{C}_{\mathrm{p} 4}$ & $\mathrm{C}_{\mathrm{p} 1} \rightarrow \mathrm{C}_{\mathrm{p} 4}$ \\
\hline$\Phi 2$ & $\mathrm{C}_{\mathrm{i}}-\mathrm{N}-\mathrm{C}_{\mathrm{p} 1}-\mathrm{C}_{\mathrm{p} 2}$ & $\mathrm{C}_{\mathrm{i}} \rightarrow \mathrm{C}_{\mathrm{p} 2}$ \\
\hline$\Phi 3$ & $\mathrm{C}_{\mathrm{p} 1}-\mathrm{C}_{\mathrm{p} 2}-\mathrm{O}-\mathrm{C}_{\mathrm{p} 3}$ & $\mathrm{C}_{\mathrm{p} 1} \rightarrow \mathrm{C}_{\mathrm{p} 3}$ \\
\hline$\Phi 4$ & $\mathrm{C}_{\mathrm{p} 1}-\mathrm{C}_{\mathrm{p} 2}-\mathrm{C}_{\mathrm{p} 3}-\mathrm{C}_{\mathrm{i}}$ & $\mathrm{C}_{\mathrm{p} 1} \rightarrow \mathrm{C}_{\mathrm{i}}$ \\
\hline$\Phi 5$ & $\mathrm{H}-\mathrm{C}_{\mathrm{p} 1}-\mathrm{C}_{\mathrm{p} 2}-\mathrm{C}_{\mathrm{p} 3}$ & $\mathrm{H} \rightarrow \mathrm{C}_{\mathrm{p} 3}$ \\
\hline
\end{tabular}



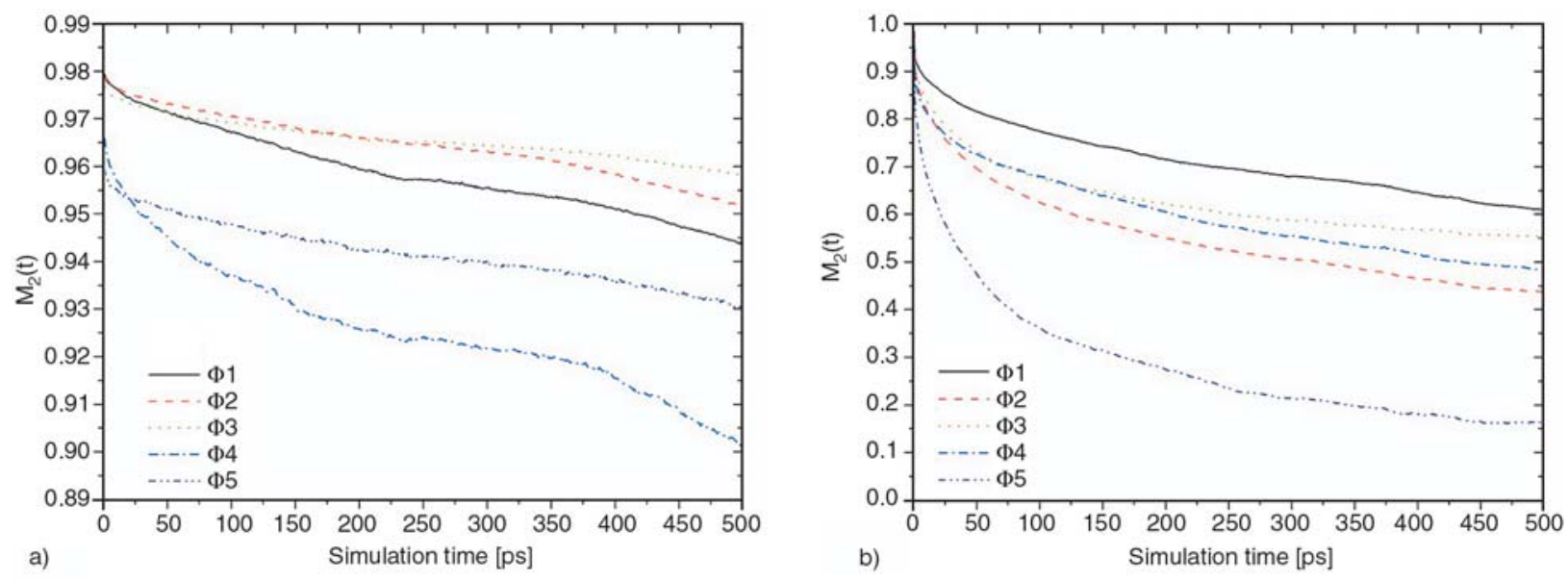

Figure 9. Vector autocorrelation functions of vector bonds corresponding to $\Phi 1, \Phi 2, \Phi 3$, $\Phi 4$ and $\Phi 5$ in 3,3',4,4'-BPDAODA with constant-NVT MD simulation at (a) $T=400 \mathrm{~K}$ and (b) $750 \mathrm{~K}$
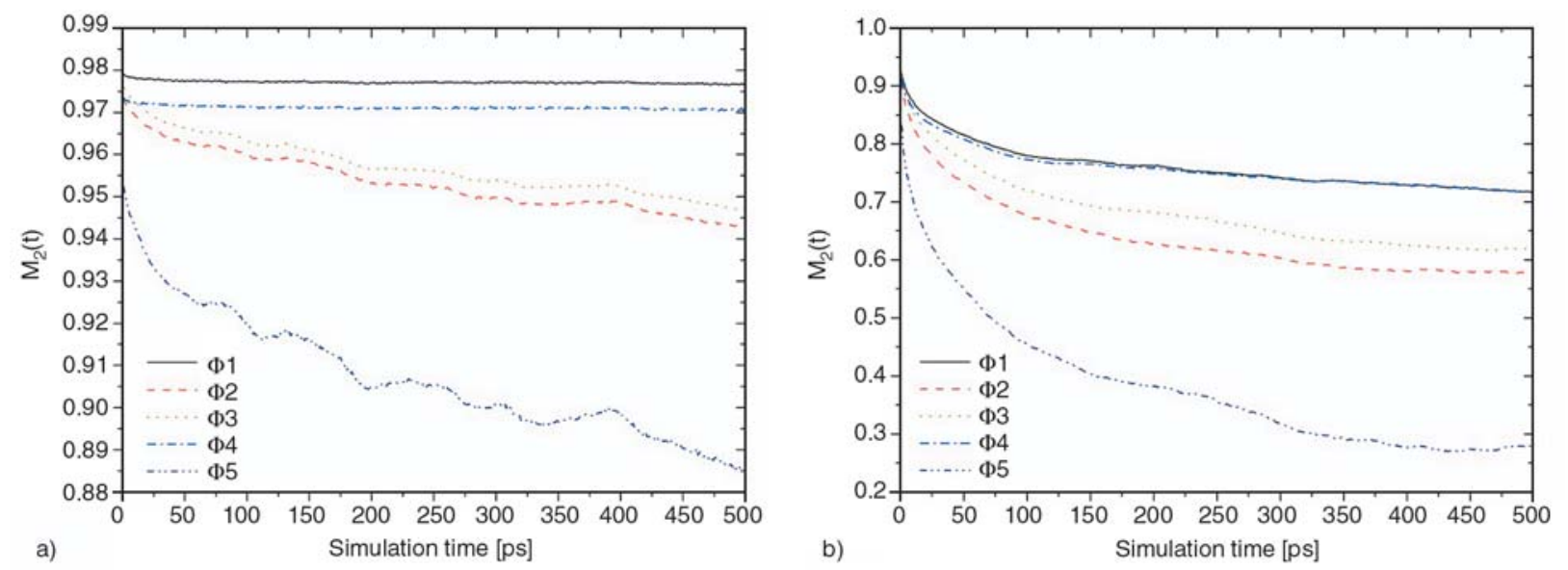

Figure 10. Vector autocorrelation functions of vector bonds corresponding to $\Phi 1, \Phi 2, \Phi 3, \Phi 4$ and $\Phi 5$ in 2,2',3,3'-BPDA-ODA with constant-NVT MD simulation at (a) $T=400 \mathrm{~K}$ and (b) $750 \mathrm{~K}$

$M_{2}(t)$ in two PIs are above 0.9 during the simulation time, indicating high chain rigidity of PIs. In 3,3',4,4'-BPDA-ODA system, the mobility of $\Phi 1$ is higher than $\Phi 2$ and $\Phi 3$, but the opposite result is obtained in 2,2',3,3'-BPDA-ODA system. The reason is that higher steric hindrance between biphenyl group can lead to lower torsion mobility of 2,2',3,3'-BPDA-ODA system. Because the aromatic rings can keep in a plane respectively during the simulation, we can define $\mathrm{C}_{\mathrm{p} 1} \rightarrow \mathrm{C}_{\mathrm{i}}(\Phi 4)$ to characterize the torsion mobility of phenyl and neighboring imide rings. Also, we can define $\mathrm{H} \rightarrow \mathrm{C}_{\mathrm{p} 3}$ (Ф5) to characterize that of phenyl ring in diamine structure. In this way, the cooperative motion among $\Phi 1, \Phi 2$ and $\Phi 3$ can be characterized through $\Phi 4$ and $\Phi 5$. In 3,3',4,4'-BPDA-ODA system, the mobility of $\Phi 4$ is much higher than $\Phi 1$ and $\Phi 2$, for it exists a positive cooperative motion between $\Phi 1$ and $\Phi 2$. Yet, in 2,2',3,3'-BPDA-ODA system, mobility of $\Phi 4$ lies between $\Phi 1$ and $\Phi 2$, which indicates that $\Phi 1$ has reverse torsion motion with $\Phi 2$, thereby it weaken the mobility of $\Phi 4$. As for $\Phi 5$, its mobility is much higher than $\Phi 2$ and $\Phi 3$ in two PI systems, showing the positive cooperative motion between $\Phi 2$ and $\Phi 3$. Possible torsion model is shown in Figure 1, and the result confirms the inference of torsion analysis via DMA method in the previous study [6]. In two PI systems, the mobility of five torsion angles shows an obvious increment at $750 \mathrm{~K}$, and the same arrangement order in mobility, that is $\Phi 5>\Phi 2>\Phi 3 \approx \Phi 4>\Phi 1$. This result indicates that the $\Phi 2$ and $\Phi 3$ are easier than $\Phi 1$ to be influenced by thermal fluctuation. The mobility of $\Phi 2$ and $\Phi 3$ increase more quickly at higher temperature, and then the conformation transitions are easier to take place. So the corresponding torsion angles are distributed wider (see Figure 5 and Figure 6 ). The mobility of $\Phi 1$ is the lowest among the five torsion angles, and hence it can be used to compare the mobility of the polymer 
chains in two PI systems. At $750 \mathrm{~K}$, the value of $M_{2}(t)$ in 2,2',3,3'-BPDA-ODA system is higher than that of $3,3^{\prime}, 4,4^{\prime}$-BPDA-ODA system. This result indicates that 2,2',3,3'-BPDA-ODA system has lower mobility than $3,3^{\prime}, 4,4^{\prime}$ '-BPDA-ODA system. And it also confirms the claim that the higher $T_{g}$ in isomeric $2,2^{\prime}, 3,3^{\prime}$-BPDA-ODA system is mainly attributed to the limited torsion motion between biphenyl groups. Yet, in two PI systems, the mobility of $\Phi 4$ lays between $\Phi 1$ and $\Phi 2$, while that of $\Phi 5$ is much higher than $\Phi 2$ and $\Phi 3$. In other words, the positive comparative motion of $\Phi 1$ and $\Phi 2$ in 3,3',4,4'-BPDA-ODA system disappears in the rubbery state. It may be resulted from partial reverse motion of diamine structure above glass transition temperature.

\section{Conclusions}

MD simulation was performed to study the glass transition temperature of isomeric PIs. Simulated $T_{g}$ is consistent with experimental value: $T_{g}$ of isomeric 2,2',3,3'-BPDA-ODA system is higher than 3,3',4,4'-BPDA-ODA system.

The glass transition temperature of polymeric materials is mainly determined by the segment motion of a polymer chain. It mainly involves the factors at space scale (free volume) and time scale (chain rigidity). Higher rigidity and less free volume lead to the higher $T_{g}$ of polymers, however, these two factors are in opposition in isomeric PI (2,2',3,3'-BPDA-ODA). The higher free volume fraction of isomeric PI can provide the polymer with more space to obtain the segment motion. However, from the torsion angle distribution calculations, it is shown that the torsion angle of its biphenyl group is constrained. Furthermore, from mean square displacement and vector autocorrelation functions calculations, this group is observed to rotate against other groups in the glassy state, and increases the chain rigidity to a great extent. So the isomeric PI needs much more relaxation time for the segment motion. Therefore, the higher $T_{g}$ of isomeric PI is mainly attributed to the chain rigidity for the time scale, not the free volume for the space scale.

\section{Acknowledgements}

We are grateful for the financial support from the National Natural Science Foundation of China (No.50433010).

\section{References}

[1] Ding M.: Isomeric polyimides. Progress in Polymer Science, 32, 623-668 (2007). DOI: $10.1016 /$ j.progpolymsci.2007.01.007

[2] Hsiao S-H., Liou G-H.: Preparation and characterization of aromatic polyamides from 4,4'-(2,6-naphthylenedioxy)dibenzoic acid and aromatic diamines. Macromolecular Chemistry and Physics, 199, 23212328 (1999).

DOI: $10.1002 /($ SICI)1521-3935(19981001)199:10 $\leq 2321::$ AID-MACP2321>3.0.CO;2-R

[3] Parthiban A., Le Guen A., Yansheng Y., Hoffmann U., Klapper M., Müllen K.: Amino-functionalized poly(arylene ether ketone)s. Macromolecules, 30, 2238-2243 (1997).

DOI: $10.1021 / \mathrm{ma} 961310 \mathrm{y}$

[4] Goodwin A. A., Campbell J. A., Wang Z. Y.: Poly(aryl ether ketone)s containing dibenzoylbiphenyl groups: Dynamic mechanical and physical ageing behaviour. Polymer International, 48, 353-359 (1999).

DOI: 10.1002/(SICI)1097-0126(199905)48:5<353::

\section{AID-PI138>3.0.CO;2-J}

[5] Tong Y., Huang W., Luo J., Ding M.: Synthesis and properties of aromatic polyimides derived from 2,2',3,3'-biphenyltetracarboxylic dianhydride. Journal of Polymer Science Part A: Polymer Chemistry, 37, 1425-1433 (1999).

DOI: $10.1002 /($ SICI $) 1099-0518(19990515) 37: 10$ $\leq 1425:$ :AID-POLA4>3.0.CO;2-G

[6] Kochi M., Chen C., Yokota R., Hasegawa M., Hergenrother P.: Isomeric biphenyl polyimides. (II) Glass transitions and secondary relaxation processes. High Performance Polymers, 17, 335-347 (2005). DOI: $10.1177 / 0954008305055557$

[7] Hasegawa M., Sensui N., Shindo Y., Yokota R.: Structure and properties of novel asymmetric biphenyl type polyimides. Homo- and copolymers and blends. Macromolecules, 32, 387-396 (1999).

DOI: $10.1021 / \mathrm{ma} 9808629$

[8] Yu K., Li Z., Sun J.: Polymer structures and glass transition: A molecular dynamics simulation study. Macromolecular Theory and Simulations, 10, 624633 (2001).

DOI: 10.1002/1521-3919(20010701)10:6<624::AIDMATS624>3.0.CO;2-K

[9] Han J., Gee R. H., Boyd R. H.: Glass transition temperatures of polymers from molecular dynamics simulations. Macromolecules, 27, 7781-7784 (1994). DOI: $10.1021 / \mathrm{ma} 00104 \mathrm{a} 036$ 
[10] Pozuelo J., Baselga J.: Glass transition temperature of low molecular weight poly(3-aminopropyl methyl siloxane). A molecular dynamics study. Polymer, 43, 6049-6055 (2002).

DOI: $10.1016 / \mathrm{S} 0032-3861(02) 00442-1$

[11] Zhang J., Liang Y., Yan J., Lou J.: Study of the molecular weight dependence of glass transition temperature for amorphous poly(L-lactide) by molecular dynamics simulation. Polymer, 48, 4900-4905 (2007).

DOI: $10.1016 /$ j.polymer.2007.06.030

[12] Wu C., Xu W.: Atomistic molecular simulations of structure and dynamics of crosslinked epoxy resin. Polymer, 48, 5802-5812 (2007).

DOI: $10.1016 /$ j.polymer.2007.07.019

[13] Liang T., Yang X., Zhang X.: Prediction of polyimide materials with high glass-transition temperatures. Journal of Polymer Science Part B: Polymer Physics, 39, 2243-2251 (2001).

DOI: $10.1002 /$ polb.1198

[14] Yani Y., Lamm M. H.: Molecular dynamics simulation of mixed matrix nanocomposites containing polyimide and polyhedral oligomeric silsesquioxane (POSS). Polymer, 50, 1324-1332 (2009). DOI: $10.1016 /$ j.polymer.2008.12.045

[15] Materials Studio 4.0 software: http://www.accelrys.com

[16] Mayo S. L., Olafson B. D., Goddard W. A.: DREIDING: A generic force field for molecular simulations. The Journal of Physical Chemistry, 94, 8897-8909 (1990).

DOI: $10.1021 / \mathrm{j} 100389 \mathrm{a} 010$

[17] Theodorou D. N., Suter U. W.: Detailed molecular structure of a vinyl polymer glass. Macromolecules, 18, 1467-1478 (1985). DOI: $10.1021 / \mathrm{ma00149a018}$

[18] Theodorou D. N., Suter U. W.: Atomistic modeling of mechanical properties of polymeric glasses. Macromolecules, 19, 139-154 (1986). DOI: $\underline{10.1021 / \mathrm{ma} 00155 \mathrm{a} 022}$
[19] Pan R., Liu X., Zhang A., Gu Y.: Molecular simulation on structure-property relationship of polyimides with methylene spacing groups in biphenyl side chain. Computational Materials Science, 39, 887-895 (2007).

DOI: $10.1016 /$ j.commatsci.2006.10.019

[20] Nosé S.: A molecular dynamics method for simulations in the canonical ensemble. Molecular Physics, 52, 255-268 (1984).

DOI: $10.1080 / 00268978400101201$

[21] Nosé S.: A unified formulation of the constant temperature molecular dynamics methods. The Journal of Chemical Physics, 81, 511-519 (1984).

DOI: $10.1063 / 1.447334$

[22] Berendsen H. J. C., Postma J. P. M., van Gunsteren W. F., DiNola A., Haak J. R.: Molecular dynamics with coupling to an external bath. The Journal of Chemical Physics, 81, 3684-3690 (1984).

DOI: $10.1063 / 1.448118$

[23] Cypcar C. C., Camelio P., Lazzeri V., Mathias J. L., Waegell B.: Prediction of the glass transition temperature of multicyclic and bulky substituted acrylate and methacrylate polymers using the energy, volume, mass (EVM) QSPR model. Macromolecules, 29, 8954-8959 (1996).

DOI: $10.1021 / \mathrm{ma961170s}$

[24] Camelio P., Lazzeri V., Waegell B., Cypcar C., Mathias J. L.: Glass transition temperature calculations for styrene derivatives using the energy, volume, and mass model. Macromolecules, 31, 2305-2311 (1998). DOI: $10.1021 / \mathrm{ma970983t}$

[25] Zhang J., Lu J., Liu W., Xue Q.: Separation of $\mathrm{CO}_{2}$ and $\mathrm{CH}_{4}$ through two types of polyimide membrane. Thin Solid Films, 340, 106-109 (1999). DOI: 10.1016/S0040-6090(98)01350-9

[26] Yang H., Li Z-S., Qian H., Yang Y., Zhang X., Sun C.: Molecular dynamics simulation studies of binary blend miscibility of poly(3-hydroxybutyrate) and poly(ethylene oxide). Polymer, 45, 453-457 (2004). DOI: 10.1016/j.polymer.2003.11.021 